\title{
Single transseptal puncture technique and contact force catheter: A simplified ablation strategy for paroxysmal atrial fibrillation
}

\author{
ZHONGPENG DU, FENG HU, LINGMIN WU, LIHUI ZHENG, \\ LIGANG DING, ERPENG LIANG, GANG CHEN and YAN YAO

\begin{abstract}
Cardiac Arrhythmia Center, Fuwai Hospital, National Center for Cardiovascular Diseases, Chinese Academy of Medical Sciences and Peking Union Medical College, Xicheng, Beijing 100037, P.R. China
\end{abstract}

Received November 4, 2019; Accepted July 1, 2020

DOI: $10.3892 /$ etm.2020.9087

\begin{abstract}
The present study aimed to evaluate the safety and efficacy of an optimized single transseptal puncture technique and contact force sensing atrial fibrillation (AF) radiofrequency catheter ablation (RFCA) strategy within a clinical setting. Fast anatomic mapping and contact force sensing ablation was applied to patients with paroxysmal AF (PAF) ablation between September 2014 and December 2016 using a single trans-septal sheath. Pulmonary vein isolation (PVI) and linear ablation were performed in PAF individually with a 10-20 g contact force with minimal fluoroscopy. Stimulation with $10 \mathrm{~mA}$ outputs on the lesions without capture was used as endpoint. A total of 419 consecutive patients who underwent first-time RFCA were enrolled in the current study, and acute PVI was achieved in all patients. The average procedure time was $74.5 \pm 9.7 \mathrm{~min}$, with an average ablation time of $27.3 \pm 7.8 \mathrm{~min}$. The average fluoroscopy time was $4.7 \pm 3.3 \mathrm{~min}$ and the average radiation dose was $24.3 \pm 25.2 \mathrm{mGy}$. At a mean follow-up time of $14.5 \pm 4.1$ months, sinus rhythm was maintained at $85.0 \%$. Cardiac tamponade occurred in one case. The results indicated that this simplified technique was a simple, safe and effective approach for PAF ablation therapy.
\end{abstract}

\section{Introduction}

Radiofrequency catheter ablation (RFCA) is used as first-line therapy in selected patients with drug-refractory symptomatic atrial fibrillation (AF) $(1,2)$. Ablation strategies that target the pulmonary vein antra are the cornerstone for the majority of AF ablation procedures (3-5). Twice transseptal punctures with

Correspondence to: Dr Yan Yao, Cardiac Arrhythmia Center, Fuwai Hospital, National Center for Cardiovascular Diseases, Chinese Academy of Medical Sciences and Peking Union Medical College, 167 Beilishi Road, Xicheng, Beijing 100037, P.R. China E-mail: ianyao@263.net.cn

Key words: atrial fibrillation, catheter ablation, contact-force sensing, fast anatomical mapping ablating and monitoring pulmonary venous potential simultaneously are commonly applied in the vast majority of cardiac electrophysiology centers $(6,7)$. However, the traditional mapping and ablation techniques without real-time contact force sensing show poor efficiency on permanent transmural lesion formation and may lead to excessive X-ray exposure and procedure time. Persistent iatrogenic atrial septal defect after transseptal puncture has been observed and complications associated with septal puncture may also be increased, as this technique punctures more than one site in fossa ovalis, particularly in complicated cases (8).

To improve the effectiveness and safety with reducing fluoroscopy of the AF ablation procedure, a simplified ablation strategy was developed that combines the single transseptal puncture technique, fast anatomical mapping (FAM) of the left atrium (LA), a contact force (CF) sensing catheter, and the high output stimulation verification technique (9). The present study aimed to demonstrate the value of this ablation strategy for patients with paroxysmal AF (PAF).

\section{Patients and methods}

Patient selection. A total of 419 PAF patients with non-valvular, antiarrhythmic drug refractory PAF who underwent de novo RFCA at Fuwai Hospital between September 2014 and December 2016 were prospectively enrolled in the present study. These patients were diagnosed with PAF according to the standard clinical guidelines (10). The present study was approved by the Institutional Ethics Committee for Biomedical Research of Fuwai Hospital and registered at Chinese Clinical Trial Registry (Unique identifier: ChiCTR2000033663). Written informed consent was obtained from each patient. Patients with non-valvular, antiarrhythmic drug refractory PAF diagnosed according to the standard clinical guidelines were included in the present study. Patients who exhibited a previous AF ablation history, LA size $>55 \mathrm{~mm}$ measured by echocardiogram, documented LA thrombus, severe pulmonary diseases, or previous cardiac surgical history were excluded from the present study. The details of their clinical characteristics are presented in Table I. There were 275 male patients $(65.6 \%)$ and the average age was $58.7 \pm 10.9$ years old. 
Table I. Baseline characteristics of patients $(n=419)$.

\begin{tabular}{lc}
\hline Characteristics & Value $(\%)$ \\
\hline Age (years) & $58.7 \pm 10.9$ \\
Sex & \\
Male & $275(65.6 \%)$ \\
Duration of AF (years) & $4.1 \pm 4.3$ \\
Patients with $>1$ year of AF & $267(63.7 \%)$ \\
CHA2DS2-VASc score & \\
0 & $90(21.5 \%)$ \\
1 & $131(31.3 \%)$ \\
2 & $107(25.5 \%)$ \\
3 & $54(12.9 \%)$ \\
4 & $23(5.5 \%)$ \\
5 & $12(2.9 \%)$ \\
6 & $2(0.5 \%)$ \\
Diabetes & $56(13.4 \%)$ \\
Heart failure & $1(0.2 \%)$ \\
Hypertension & $199(47.5 \%)$ \\
Myocardial infarction & $10(2.4 \%)$ \\
Peripheral vascular disease & $9(2.0 \%)$ \\
History of stroke & $26(6.2 \%)$ \\
LVEF (\%) & $63.9 \pm 6.6$ \\
LA size (mm) & $36.5 \pm 4.8$ \\
\hline
\end{tabular}

AF, atrial fibrillation; LVEF, left ventricular ejection fraction; LA, left atrial.

Simplified electrophysiological procedures. All procedures were conducted under conscious sedation, and catheters were typically inserted via the right femoral vein. After positioning the coronary sinus catheter as an anatomical landmark, the transseptal puncture was performed under fluoroscopy using a single $8.5 \mathrm{Fr}$ sheath only (11). FAM of the LA was guided by the CARTO ${ }^{\circledR} 3$ system (Biosense Webster, Inc.) using a PentaRay catheter (Biosense Webster, Inc.). At this stage, a CF catheter (THERMOCOOL SMARTTOUCH ${ }^{\circledast}$ Catheter; Biosense Webster, Inc.) was out of the body but with the tip placed at the cardiac silhouette of the chest (Fig. 1).

The PentaRay catheter was taken off the sheath when FAM of LA was accomplished and the CF catheter was inserted into the LA. Circumferential pulmonary vein isolation (CPVI) was performed in the present study. The maximal power and temperature were set as $40 \mathrm{~W}$ and $43^{\circ} \mathrm{C}$, respectively. The catheter was continuously irrigated with saline at a speed of $17 \mathrm{ml} / \mathrm{min}$ and the $\mathrm{CF}$ was maintained between 10 and $20 \mathrm{~g}$ during the ablation procedure. Ablation tags were annotated with the CARTO VISITAG ${ }^{\mathrm{TM}}$ Module (Biosense Webster, Inc.).

To verify PVI, stimulation with $10 \mathrm{~mA}$ outputs along the ablation lesions was delivered through the distal electrode of the ablation catheter. Additional ablation was performed if conduction gaps were identified. A successful procedure was defined by the absence of LA capture at all pacing sites (Fig. 2).

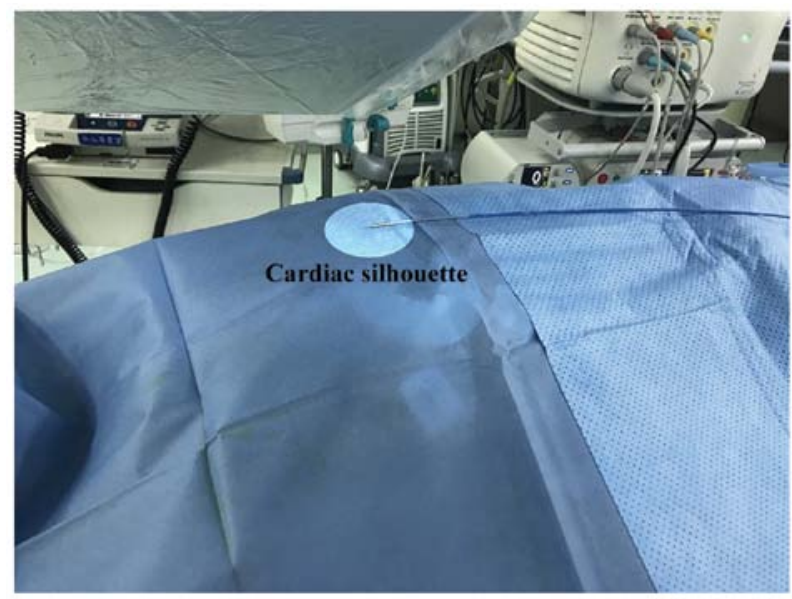

Figure 1. Placement of ablation catheter during left atrial mapping.

Post-ablation follow-up. Antiarrhythmic medications, including propafenone and amiodarone, were administered for 3 months after ablation in all patients, then terminated if no AF recurred. An electrocardiogram (ECG) and $24 \mathrm{~h}$ Holter were obtained at 1, 3, 6,9 and 12 months post-ablation during the follow-up. An additional ECG and Holter were also performed if symptoms suggestive of AF recurrence occurred. After the 3 month blanking period, arrhythmia recurrence was defined as any episode ( $>30 \mathrm{sec}$ duration) of AF or atrial tachycardia (AT).

Study endpoints. The primary effectiveness endpoint was freedom from any documented episode of AF/AT, which sustained for $>30$ sec during the 12 month follow up and outside a blanking period of 3 months. Secondary endpoints included procedure time and ablation time, procedure-related complications, and repeated ablation procedure during follow-up.

Statistical analysis. Continuous data were summarized as mean \pm standard deviation. Categorical data were summarized as counts and percentages. Comparisons of categorical variables were performed using $\chi^{2}$ tests. Rates of survival from atrial arrhythmia recurrence following the 3 month blanking period were estimated with a Kaplan-Meier model. Cox regression models were used to test for the significance of patient baseline characteristics and procedural detail in predicting atrial arrhythmia recurrence rates, as well as for calculating hazard ratios (HRs) to compare recurrence risks. All statistical analyses were performed using SPSS v23.0 software (IBM Corp.).

\section{Results}

Procedural parameters. The procedural parameters are summarized in Table II. Entrance/exit block in all PVs during the procedure were achieved in $415(99.0 \%)$ patients. The average procedure time was $74.5 \pm 9.7 \mathrm{~min}$ and the average ablation time was $27.3 \pm 7.8 \mathrm{~min}$. In addition, the average radiation dose was $24.3 \pm 25.2 \mathrm{mGy}$.

Follow-up for effectiveness. At a mean follow-up time of $14.5 \pm 4.1$ months, $18(4.3 \%)$ patients were unable to be contacted, including one patient who died due to pulmonary 


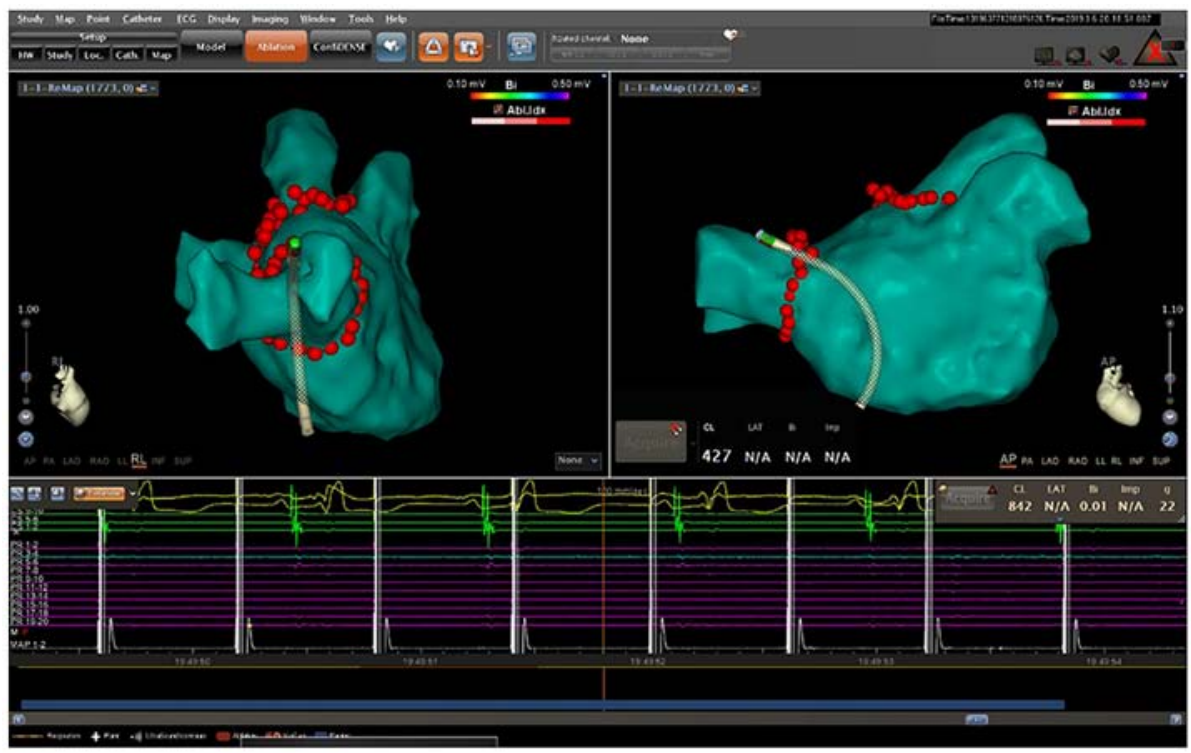

Figure 2. Verification of pulmonary vein isolation with high output stimulation. Stimulation with $10 \mathrm{~mA}$ outputs along the ablation lesions (red point) was delivered through the distal electrode of the ablation catheter. Due to successful pulmonary vein isolation, the atrial rhythm was not related to pacing rhythm.

Table II. Procedural and complication data.

\begin{tabular}{lc}
\hline Factor & Value \\
\hline Procedure time (min) & $74.5 \pm 9.7$ \\
Ablation time (min) & $27.3 \pm 7.8$ \\
Fluoroscopy time (min) & $4.7 \pm 3.3$ \\
PVI ablation (\%) & $419(100.0)$ \\
Acute procedural success (\%) & $415(99.0)$ \\
AF persisted after ablation (\%) & $0(0.0)$ \\
Acute PVI reconnection (\%) & $0(0.0)$ \\
Complications & $5(1.2)$ \\
Pericardial effusion & $1(0.2)$ \\
Arteriovenous fistulas & $2(0.5)$ \\
Femoral artery pseudoaneurysm & $2(0.5)$
\end{tabular}

$\mathrm{PVI}$, pulmonary vein isolation; $\mathrm{AF}$, atrial fibrillation.

carcinoma without AF recurrence. Kaplan-Meier analysis estimated that 341 (85.0\%) patients were free from AF/AT during follow-up (Fig. 3). A total of 7 patients underwent repeat ablation procedures during follow-up. Electric reconduction of PVI was demonstrated during the repeat procedures, and re-ablation at gaps were performed.

Multivariable Cox regression modeling demonstrated that the duration of $\mathrm{AF}$ was a significant predictor of recurrence (Table III). The greatest risk was an $\mathrm{AF}$ duration $>1$ year, relative to a duration of $\leq 1$ year [HR, 2.0; 95\% confidence interval (CI), 1.2-3.2]. A history of hypertension resulted in a reduced risk, as evidenced by a HR of <1 (HR, 0.6; 95\% CI, 0.4-0.9).

Complications. The overall procedure-related complication rate was $1.2 \%$, including $1(0.2 \%)$ case of pericardial effusion and $4(1.0 \%)$ cases of vascular access complications. A total of 2 cases of arteriovenous fistulas were resolved with

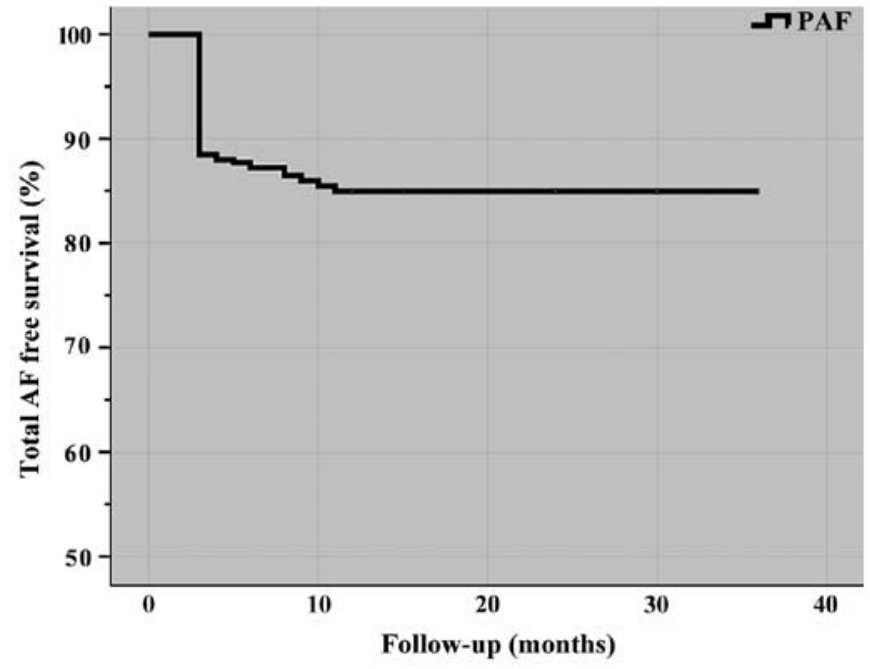

Figure 3. Follow-up outcomes of AF free survival. PAF, paroxysmal atrial fibrillation; $\mathrm{AF}$, atrial fibrillation.

only conservative medical therapy. In addition, 1 case of pericardial effusion required pericardiocentesis and 2 cases of femoral artery pseudoaneurysm required puncture, suction and compression. There were no strokes during the ablation visit or follow-ups (Table II).

\section{Discussion}

The present study demonstrated the advantages of a simplified ablation procedure for PAF of combined single transseptal puncture, FAM of LA, CF-sensing ablation and the high output stimulation verification technique among a large number of patients with PAF. In the current study, the 12 month AF/AT-free survival rate was improved compared with previous studies $(12,13)$, while the average procedure time was just $74.5 \pm 9.7 \mathrm{~min}$ and the complication rate was controlled at a considerably lower level, which suggests this 
Table III. Cox regression predictors of recurrence after a 3 month blanking period.

Hazard Ratio

\begin{tabular}{lcc}
\cline { 2 - 3 } Parameter comparison & $(95 \% \mathrm{CI})$ & $\chi^{2} \mathrm{P}$-value \\
\hline Duration of AF (>1 year vs. s1 year) & $3.0(1.5,5.9)$ & 0.002 \\
History of hypertension (Yes vs. no) & $0.7(0.4,1.2)$ & 0.264 \\
History of diabetes (Yes vs. no) & $1.6(0.8,3.1)$ & 0.145 \\
Left atrial size (per mm increase) & $1.0(1.0,1.1)$ & 0.935
\end{tabular}

$\mathrm{n}=415$ patients with non-missing values for all included variables. $\mathrm{AF}$, atrial fibrillation; CI, confidence interval.

simplified and practical strategy is beneficial in a clinical setting. The multiple-factor analysis demonstrated that the duration of AF and left atrial size were significant predictors of recurrence, whereas the history of hypertension resulted in a reduced risk. Although this finding may initially appear counter-intuitive, it is supported by a prior study and is likely due to the protective effect of medications used to treat hypertension (14).

Radiofrequency ablation for patients with AF generally requires two transseptal punctures to deliver a multipolar mapping catheter and an ablation catheter into the left atrium, respectively. However, this procedure requires a skilled operator to perform it, and in most cases intracardiac echocardiography is required (8). Puncture-related complications and iatrogenic atrial septal defects are increased followed by an increase in the number of punctures (8). In a previous study, a modified transseptal puncture protocol was developed that used only a coronary sinus catheter as the landmark under fluoroscopy (11). In the present study, all transseptal procedures were overwhelmingly accomplished by fellows and guided only by fluoroscopy.

A number of different parameters are known to affect the transmurality of ablation lesions, including catheter tip temperature, power output, ablation time and CF. It has previously been demonstrated that real-time electrogram amplitude and impedance are poor predictors of the true CF applied (6). It is important to have an accurate measure of CF because a higher CF may increase the risk of blood charring (15). CF-guided catheters can provide stable and moderate $\mathrm{CF}$, allowing for improvements in ablation safety and effectiveness, while simultaneously reducing procedure and fluoroscopy times (16). The improved catheter stability leads to faster transmural lesion formation (17), particularly in the right side PV (18). Procedures have been shortened due to faster assessment of appropriate catheter contact, resulting in the reduction of radiation (14,19-21). In CF-guided PV isolation, pulmonary vein reconnection remains primarily attributable to insufficient lesion depth and contiguity (17). Additionally, since the achievement of ideal ablation lesions depends on a combination of $\mathrm{CF}$, power and duration parameters, the integration of these parameters via an automated algorithm, such as the Visitag with Ablation Index, may provide a valuable solution to this complex optimization problem (22-24).

FAM, which is guided by a three-dimensional (3D) mapping system and a circular or multi-electrode mapping catheter, also serve a role in CF-guided ablation (25). Traditional point-to-point modeling cannot rapidly and accurately map the true LA geometry; therefore, it typically leads to increased fluoroscopy usage in order to reduce the complication risk (25). Alternatively, FAM can provide precise LA modeling and electronic substrate mapping information, leading to fewer manipulation difficulties and lower radiation $(8,26)$. FAM guidance has been indicated to allow procedures with nearly zero fluoroscopy and without compromising the procedure duration, effectiveness or safety $(25,27)$.

High output stimulation provides a convenient and reliable approach for the verification of ablation lesions. The traditional endpoint of PVI is antral disconnection detected by a circular mapping catheter, which requires complex catheter manipulation to ensure sufficient contact (22). High output stimulation along the encircling lesion line without LA capture could also effectively vivificate the conduction block between all PVs and LA (28). Guided with 3D mapping and CF monitoring, an operator can ensure ablation line integrity without concerns regarding poor contact or inaccurate location $(9,29)$. Supplementary ablation to touch up any residual gaps (LA capture during high output stimulation along the lesion line) can be performed immediately, thus decreasing the procedure and fluoroscopy times.

There are some limitations of the present study. Firstly, the current study reflects the experience of a single center in China, and thus may not be representative of results across sites with differing workflows, levels of operator experience or patient populations. Secondly, the current study did not set a control group with the twice transseptal puncture. Giving the low complication rates and acceptable sinus rhythm maintenance during follow-up, the choice of this simplified strategy is also a reasonable option. Additionally, atrial arrhythmia recurrence estimates could potentially be biased due to patients with an incomplete follow-up, although the magnitude of this bias could not be significant due to the low number of these patients with $<12$ months of follow-up.

In conclusion, the present study demonstrated that this simplified technique was a simple, safe and effective approach for PAF ablation therapy. This strategy is a reasonable alternative for patients experiencing difficulty undergoing twice septal puncture.

\section{Acknowledgements}

Not applicable.

\section{Funding}

No funding was received.

\section{Availability of data and materials}

The datasets used and/or analyzed during the current study are available from the corresponding author on reasonable request. 


\section{Authors' contributions}

YY conceived and designed the study. ZD, LZ, LD, EL and GC conducted the research and acquired the data. FH and LW analyzed and interpreted the data. ZD drafted the manuscript. All authors substantially contributed to the revision of the manuscript, and approved the final version.

\section{Ethics approval and consent to participate}

The current study was approved by the Institutional Ethics Committee for Biomedical Research of Fuwai Hospital and registered at Chinese Clinical Trial Registry (Unique identifier: ChiCTR2000033663).

\section{Patient consent for publication}

Not applicable.

\section{Competing interests}

The authors declare that they have no competing interests.

\section{References}

1. Kirchhof P, Benussi S, Kotecha D, Ahlsson A, Atar D, Casadei B, Castella M, Diener HC, Heidbuchel H, Hendriks J, et al; ESC Scientific Document Group: 2016 ESC Guidelines for the management of atrial fibrillation developed in collaboration with EACTS. Eur Heart J 37: 2893-2962, 2016.

2. January CT, Wann LS, Alpert JS, Calkins H, Cigarroa JE, Cleveland JC Jr, Conti JB,EllinorPT,Ezekowitz MD,FieldME, etal; ACC/AHA Task Force Members: 2014 AHA/ACC/HRS guideline for the management of patients with atrial fibrillation: A report of the American College of Cardiology/American Heart Association Task Force on practice guidelines and the Heart Rhythm Society. Circulation 130: e199-e267, 2014.

3. Haïssaguerre M, Shah DC, Jaïs P, Hocini M, Yamane T, Deisenhofer I, Chauvin M, Garrigue S and Clémenty J: Electrophysiological breakthroughs from the left atrium to the pulmonary veins. Circulation 102: 2463-2465, 2000.

4. Verma A, Jiang CY, Betts TR, Chen J, Deisenhofer I, Mantovan R, Macle L, Morillo CA, Haverkamp W, Weerasooriya R, et al; STAR AF II Investigators: Approaches to catheter ablation for persistent atrial fibrillation. N Engl J Med 372: 1812-1822, 2015.

5. Haïssaguerre M, Jaïs P, Shah DC, Takahashi A, Hocini M Quiniou G, Garrigue S, Le Mouroux A, Le Métayer P and Clémenty J: Spontaneous initiation of atrial fibrillation by ectopic beats originating in the pulmonary veins. N Engl J Med 339: 659-666, 1998

6. Nakagawa H,Kautzner J, Natale A,Peichl P, Cihak R, Wichterle D, Ikeda A, Santangeli P, Di Biase L and Jackman WM: Locations of high contact force during left atrial mapping in atrial fibrillation patients: Electrogram amplitude and impedance are poor predictors of electrode-tissue contact force for ablation of atrial fibrillation. Circ Arrhythm Electrophysiol 6: 746-753, 2013.

7. De Potter T, Van Herendael H, Balasubramaniam R, Wright M, Agarwal SC, Sanders P, Khaykin Y, Latcu CG, Maury P, Pani A, et al: Safety and long-term effectiveness of paroxysmal atrial fibrillation ablation with a contact force-sensing catheter: Real-world experience from a prospective, multicentre observational cohort registry. Europace 20(FI_3): f410-f418, 2018.

8. Fagundes RL, Mantica M, De Luca L, Forleo G, Pappalardo A, Avella A,Fraticelli A, Dello Russo A, Casella M,Pelargonio G, et al: Safety of single transseptal puncture for ablation of atrial fibrillation: Retrospective study from a large cohort of patients. J Cardiovase Electrophysiol 18: 1277-1281, 2007.

9. Steven D, Reddy VY, Inada K, Roberts-Thomson KC, Seiler J, Stevenson WG and Michaud GF: Loss of pace capture on the ablation line: A new marker for complete radiofrequency lesions to achieve pulmonary vein isolation. Heart Rhythm 7: 323-330, 2010.
10. Calkins H, Hindricks G, Cappato R, Kim YH, Saad EB Aguinaga L, Akar JG, Badhwar V, Brugada J, Camm J, et al: $2017 \mathrm{HRS} / \mathrm{EHRA}$ /ECAS/APHRS/SOLAECE expert consensus statement on catheter and surgical ablation of atrial fibrillation. Heart Rhythm 14: e275-e444, 2017.

11. Yao Y, Ding L, Chen W, Guo J, Bao J, Shi R, Huang W, Zhang S and Wong T: The training and learning process of transseptal puncture using a modified technique. Europace 15: 1784-1790, 2013.

12. Natale A, Reddy VY, Monir G, Wilber DJ, Lindsay BD, McElderry HT, Kantipudi C, Mansour MC, Melby DP, Packer DL, et al: Paroxysmal AF catheter ablation with a contact force sensing catheter: Results of the prospective, multicenter SMART-AF trial. J Am Coll Cardiol 64: 647-656, 2014.

13. Ullah W, McLean A, Tayebjee MH, Gupta D, Ginks MR, Haywood GA, O'Neill M, Lambiase PD, Earley MJ and Schilling RJ; UK Multicentre Trials Group ${ }^{* *}$ : Randomized trial comparing pulmonary vein isolation using the SmartTouch catheter with or without real-time contact force data. Heart Rhythm 13: 1761-1767, 2016.

14. Widimsky J: Arterial hypertension and atrial fibrillation: Selecting antihypertensive therapy. Cor Vasa 54: e248-e252, 2012.

15. Makimoto H, Metzner A, Tilz RR, Lin T, Heeger CH, Rillig A, Mathew S, Lemeš C, Wissner E, Kuck KH and Ouyang F: Higher contact force, energy setting, and impedance rise during radiofrequency ablation predicts charring: New insights from contact force-guided in vivo ablation. J Cardiovasc Electrophysiol 29: 227-235, 2018.

16. Lin H, Chen YH, Hou JW, Lu ZY, Xiang Y and Li YG: Role of contact force-guided radiofrequency catheter ablation for treatment of atrial fibrillation: A systematic review and meta-analysis. J Cardiovasc Electrophysiol 28: 994-1005, 2017.

17. Bun SS, Ayari A, Latcu DG, Errahmouni A and Saoudi N: Radiofrequency catheter ablation of atrial fibrillation: Electrical modification suggesting transmurality is faster achieved with remote magnetic catheter in comparison with contact force use. J Cardiovasc Electrophysiol 28: 745-753, 2017.

18. Nair GM, Yeo C, MacDonald Z, Ainslie MP, Alqarawi WA, Nery PB, Redpath CJ, Sadek M, Spence S, Green MS, et al: Three-year outcomes and reconnection patterns after initial contact force guided pulmonary vein isolation for paroxysmal atrial fibrillation. J Cardiovasc Electrophysiol 28: 984-993, 2017.

19. Pedrote A, Arana-Rueda E, Arce-León A, Acosta J, Gómez-Pulido F, Martos-Maine JL, Frutos-López M, Sánchez-Brotons J and García-Riesco L: Impact of Contact Force Monitoring in Acute Pulmonary Vein Isolation Using an Anatomic Approach. A Randomized Study. Pacing Clin Electrophysiol 39: 361-369, 2016.

20. El Haddad M, Taghji P, Phlips T, Wolf M, Demolder A, Choudhury R, Knecht S, Vandekerckhove Y, Tavernier R, Nakagawa H, et al: Determinants of Acute and Late Pulmonary Vein Reconnection in Contact Force-Guided Pulmonary Vein Isolation: Identifying the Weakest Link in the Ablation Chain. Circ Arrhythm Electrophysiol 10: e004867, 2017.

21. Marijon E, Fazaa S, Narayanan K, Guy-Moyat B, Bouzeman A, Providencia R, Treguer F, Combes N, Bortone A, Boveda S, et al: Real-time contact force sensing for pulmonary vein isolation in the setting of paroxysmal atrial fibrillation: Procedural and 1-year results. J Cardiovasc Electrophysiol 25: 130-137, 2014.

22. Tanaka N, Inoue K, Tanaka K, Toyoshima Y, Oka T, Okada M, Inoue H, Nakamaru R, Koyama Y,Okamura A, et al: Automated Ablation Annotation Algorithm Reduces Re-conduction of Isolated Pulmonary Vein and Improves Outcome After Catheter Ablation for Atrial Fibrillation. Circ J 81: 1596-1602, 2017.

23. Hussein A, Das M, Chaturvedi V, Asfour IK, Daryanani N, Morgan M, Ronayne C, Shaw M, Snowdon R and Gupta D: Prospective use of Ablation Index targets improves clinical outcomes following ablation for atrial fibrillation. J Cardiovasc Electrophysiol 28: 1037-1047, 2017.

24. Das M, Loveday JJ, Wynn GJ, Gomes S, Saeed Y, Bonnett LJ, Waktare JEP, Todd DM, Hall MCS, Snowdon RL, et al: Ablation index, a novel marker of ablation lesion quality: Prediction of pulmonary vein reconnection at repeat electrophysiology study and regional differences in target values. Europace 19: 775-783, 2017. 
25. Reddy VY, Morales G, Ahmed H, Neuzil P, Dukkipati S, Kim S, Clemens J and D'Avila A: Catheter ablation of atrial fibrillation without the use of fluoroscopy. Heart Rhythm 7: 1644-1653, 2010.

26. Voskoboinik A, Kalman ES, Savicky Y, Sparks PB, Morton JB, Lee G, Kistler PM and Kalman JM: Reduction in radiation dose for atrial fibrillation ablation over time: A 12-year single-center experience of 2344 patients. Heart Rhythm 14: 810-816, 2017.

27. Bulava A, Hanis J and Eisenberger M: Catheter ablation of atrial fibrillation using zero-fluoroscopy technique: A randomized trial. Pacing Clin Electrophysiol 38: 797-806, 2015.

28. Pambrun T, Combes S, Sousa P, Bloa ML, El Bouazzaoui R, Grand-Larrieu D, Thompson N, Martin R, Combes N, Boveda S, et al: Contact-force guided single-catheter approach for pulmonary vein isolation: Feasibility, outcomes, and cost-effectiveness. Heart Rhythm 14: 331-338, 2017.
29. Eitel C, Hindricks G, Sommer P, Gaspar T, Kircher S, Wetzel U, Dagres N, Esato M, Bollmann A, Husser D, et al: Circumferential pulmonary vein isolation and linear left atrial ablation as a single-catheter technique to achieve bidirectional conduction block: The pace-and-ablate approach. Heart Rhythm 7: 157-164, 2010.

This work is licensed under a Creative Commons Attribution-NonCommercial-NoDerivatives 4.0 International (CC BY-NC-ND 4.0) License. 